\title{
Novel Hemizygous IL2RG p.(Pro58Ser) Mutation Impairs IL-2 Receptor Complex Expression on Lymphocytes Causing X-Linked Combined Immunodeficiency
}

\author{
Elina A. Tuovinen ${ }^{1,2,3}$ • Juha Grönholm ${ }^{2,3}$ • Tiina Öhman ${ }^{4}$ - Sakari Pöysti ${ }^{5}$ • Raine Toivonen ${ }^{5}$ • Anna Kreutzman ${ }^{2,6}$. \\ Kaarina Heiskanen ${ }^{3}$ • Luca Trotta $^{7}$. Sanna Toiviainen-Salo ${ }^{8}$. John M. Routes ${ }^{9}$. James Verbsky ${ }^{9}$. Satu Mustjoki ${ }^{2,6,10}$. \\ Janna Saarela ${ }^{7,11,12} \cdot$ Juha Kere ${ }^{1,13,14} \cdot$ Markku Varjosalo $^{4}$ - Arno Hänninen ${ }^{5} \cdot$ Mikko R. J. Seppänen $^{2,3}$ (D)
}

Received: 23 August 2019 / Accepted: 6 January 2020 / Published online: 19 February 2020

(C) The Author(s) 2020

\begin{abstract}
Hypomorphic IL2RG mutations may lead to milder phenotypes than X-SCID, named variably as atypical X-SCID or X-CID. We report an 11-year-old boy with a novel c. 172C>T;p.(Pro58Ser) mutation in IL2RG, presenting with atypical X-SCID phenotype. We also review the growing number of hypomorphic $I L 2 R G$ mutations causing atypical X-SCID. We studied the patient's clinical phenotype, B, T, NK, and dendritic cell phenotypes, IL2RG and CD25 cell surface expression, and IL-2 target gene expression, STAT tyrosine phosphorylation, PBMC proliferation, and blast formation in response to IL-2 stimulation, as well as proteinprotein interactions of the mutated IL2RG by BioID proximity labeling. The patient suffered from recurrent upper and lower respiratory tract infections, bronchiectasis, and reactive arthritis. His total lymphocyte counts have remained normal despite skewed T and B cells subpopulations, with very low numbers of plasmacytoid dendritic cells. Surface expression of IL2RG was reduced on his lymphocytes. This led to impaired STAT tyrosine phosphorylation in response to IL-2 and IL-21, reduced expression of IL-2 target genes in patient CD4+ T cells, and reduced cell proliferation in response to IL-2 stimulation. BioID proximity labeling showed aberrant interactions between mutated IL2RG and ER/Golgi proteins causing mislocalization of the mutated IL2RG to the ER/Golgi interface. In conclusion, IL2RG p.(Pro58Ser) causes X-CID. Failure of IL2RG plasma membrane targeting may lead to atypical X-SCID. We further identified another carrier of this mutation from newborn SCID screening, lost to closer scrutiny.
\end{abstract}

Electronic supplementary material The online version of this article (https://doi.org/10.1007/s10875-020-00745-2) contains supplementary material, which is available to authorized users.

Juha Grönholm

juha.gronholm@helsinki.fi

1 Folkhälsan Research Center, Helsinki, Finland

2 Translational Immunology Research Program, University of Helsinki, Helsinki, Finland

3 Rare Diseases Center and Pediatric Research Center, New Children's Hospital, University of Helsinki and HUS Helsinki University Hospital, Helsinki, Finland

4 Systems Biology Research Group and Proteomics Unit, Institute of Biotechnology, HiLIFE, University of Helsinki, Helsinki, Finland

5 Department of Clinical Microbiology and Immunology, Turku University Hospital and Institute of Biomedicine, University of Turku, Turku, Finland

6 Hematology Research Unit Helsinki, Helsinki University Hospital Comprehensive Cancer Center, Helsinki, Finland
Institute for Molecular Medicine Finland, HiLIFE, University of Helsinki, Helsinki, Finland

8 Department of Pediatric Radiology, HUS Medical Imaging Center, Radiology, University of Helsinki and HUS Helsinki University Hospital, Helsinki, Finland

9 Department of Pediatrics, Medical College of Wisconsin, Milwaukee, WI, USA

10 Department of Clinical Chemistry and Hematology, University of Helsinki, Helsinki, Finland

11 Department of Medical Genetics, Helsinki Central University Hospital, Helsinki, Finland

12 Centre for Molecular Medicine Norway, University of Oslo, Oslo, Norway

13 Department of Biosciences and Nutrition, Karolinska Institutet, Stockholm, Sweden

14 Stem Cells and Metabolism Research Program, University of Helsinki, Helsinki, Finland 
Keywords X-linked combined immunodeficiency diseases · severe combined immunodeficiency, atypical · interleukin receptor common gamma subunit $\cdot I L 2 R G \cdot$ endoplasmic reticulum $\cdot$ Golgi apparatus

\section{Introduction}

$\mathrm{X}$-linked severe combined immunodeficiency (X-SCID) accounts for approximately half of all the SCID cases and has an estimated incidence of 1:100000 male births [1]. It is caused by mutations in interleukin-2 receptor gamma chain $(I L 2 R G)$ gene and presents with absent or profoundly diminished peripheral T and NK cells and functionally defective B cells $[2$, 3]. IL $2 R G$ is expressed by virtually all hematopoietic cells and is shared by the receptors for interleukins (IL) 2, 4, 7, 9, 15, and 21 [4]. In these receptors, it contributes to high-affinity ligand binding and coupling with the Janus kinase 3 (JAK3) with its cytoplasmic tail. Ligand binding induces phosphorylation-mediated activation of receptor-associated JAKs (1 and 3), further leading to JAK-mediated tyrosine phosphorylation, homodimerization, and nuclear translocation of signal transducers and activators of transcription (STATs) affecting target gene expression $[5,6]$.

The lack of normal IL-4, IL-7, IL-15, and IL-21 signaling explains the classical $\mathrm{T}^{-} \mathrm{B}^{+} \mathrm{NK}^{-}$phenotype in X-SCID. IL-7 and IL-15 are required for T and NK cell development, respectively, and disturbed IL-4 and IL-21 signaling causes the intrinsic B cell defect [6-8]. IL-7 has a non-redundant role in T cell development; defective IL-7-induced signaling is the main reason for the T cell lymphopenia in X-SCID [9]. As a result, individuals with $\mathrm{X}$-SCID lack adequate $\mathrm{T}$ cell function for survival $[6,10]$. They present with early-onset respiratory tract infections, diarrhea, and failure to thrive. Without stem cell transplant or gene therapy, they usually die within the first year of life $[2,5]$.

Several cases with rare hemizygous $I L 2 R G$ mutations and milder phenotypes, like X-linked combined immunodeficiency (CID) or common variable immunodeficiency (CVID), have been reported [2, 10-13]. Caused by hypomorphic mutations, genetic reversions in the early progenitor cells, or maternal T or NK cell engraftment, these atypical or "leaky" phenotypes may display preserved and/or partially functional T and NK cell subsets [3,10,12,14-19]. Typical and atypical $\mathrm{X}$-SCID have overlapping clinical features such as recurrent bacterial and viral infections, often caused by opportunistic pathogens. However, as the atypical X-SCID patients have greater amounts of residual $\mathrm{T}$ cell function, their clinical presentation is less severe and the onset usually later when compared to the classical X-SCID [10].

We report a boy with a novel c.172C $>$ T; p.(Pro58Ser) mutation in $I L 2 R G$, with impaired expression on IL-2 receptor complex, and another boy with the same mutation caught in newborn SCID screening. Our index patient presented with an
X-CID phenotype with normal numbers of T, B, and NK cells, he suffered from recurrent respiratory tract infections, bronchiectasis, and reactive arthritis.

\section{Methods}

\section{Genetic Analyses}

DNA samples were extracted from peripheral blood and lymphocyte subpopulations sorted using standard methods. Whole-exome sequencing (WES) was performed on HiSeq 1500 Rapid run (Illumina) using a SureSelect Clinical Research Capture Exome kit (Agilent). The sequencing and data analysis were performed as previously described [20,21]. Validation of the candidate disease-causing variant and screening of familial mutations in the available family members (Fig. 1a) were conducted using PCR amplification of genomic DNA and capillary electrophoresis using the ABI3730XL DNA Analyzer and BigDye Terminator Cycle Sequencing kit (Applied Biosystems). Lymphocyte subpopulation screening for the variant was performed using PCR amplification of the genomic DNA and capillary electrophoresis using the cycle sequencing technology (dideoxy chain termination/cycle sequencing) on ABI-3730XL DNA Analyzer. Detailed processing and analysis of WES along with details for the second patient are described in the Online Resource Supplementary text.

\section{Cell isolation, surface staining, and basic immunological workup}

Cell isolation is described in the Online Resource Supplementary text. Peripheral blood mononuclear cells (PBMCs) were stained with fluorescently conjugated antihuman CD4, CD19 (BioLegend), CD3, CD14 (ImmunoTools), CD16, CD56 (BD Pharmigen), and CD8 (Miltenyi Biotech) antibodies for $30 \mathrm{~min}$ on ice. After surface staining, SYTOX Green Dead Cell Stain (Invitrogen) was added to the cells, and CD4+ and CD8+ T cells, CD19+ B cells, and CD16+CD56+ NK cells were sorted with BDInflux. Basic immunological workup was performed in an accredited laboratory. Whole-blood NK cell phenotyping and TCRV $\beta$ repertoire sequencing are described in the Online Resource Supplementary text.

Expression of IL2RG (CD132) and IL2RA (CD25) was determined from $\mathrm{CD} 4+\mathrm{T}$ cells using fluorescently conjugated anti-human CD4, CD8, CD25, CD56 (BD Biosciences), and 
a

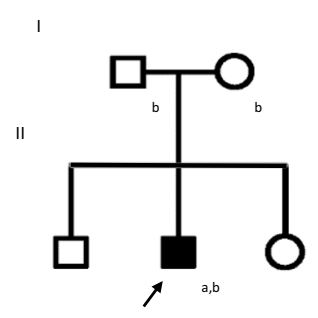

b

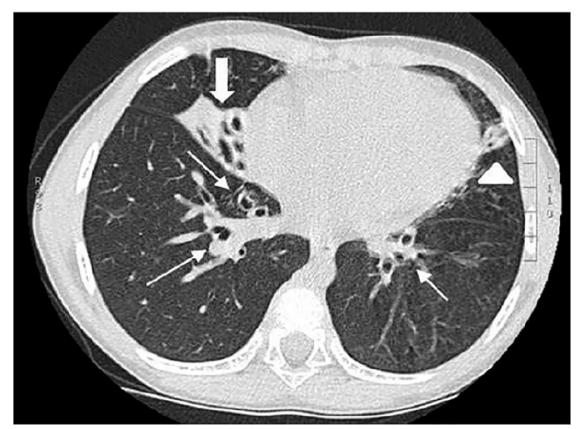

Fig. 1 Patient family pedigree, high-resolution lung computed tomography and domain structure of IL2RG. a Family pedigree ( ${ }^{\mathrm{a}}$ Whole exome sequencing; ${ }^{\mathrm{b}}$ Targeted screening by Sanger sequencing). b High-resolution lung computed tomography at age 6 years demonstrated centrally located bronchiectasis in both lower lobes (thin arrows), a cluster of cystic bronchiectasis in the completely collapsed right middle lobe (thick arrow), a fibrotic strand with small caliper traction bronchiectasis in the left lingula (arrowhead). c Schematic representation of the IL2RG Pro58Ser and previously

CD132 (eBioscience) antibodies. Briefly, antibodies were added directly to an aliquot of $100 \mu \mathrm{l}$ of freshly drawn whole blood, pre-cooled to $+4{ }^{\circ} \mathrm{C}$. After 15-min incubation, red blood cells were lysed (BD FACS Lysing Solution) and cells were analyzed by flow cytometry (NovoCyte model 3000 and NovoExpress, Acea).

\section{STAT Phosphorylation in Response to Exogenous IL-2 and IL-21}

STAT5 and STAT3 phosphorylation were measured from isolated PBMCs after a 15-min stimulation in the presence of exogenous IL-2 $(10 \mathrm{U} / \mathrm{ml}$ and $320 \mathrm{U} / \mathrm{ml})$ or IL-21 $(10 \mathrm{ng} / \mathrm{ml})$, respectively, in pre-warmed RPMI 1640. IL-2-stimulated cells were then fixed and permeabilized according to manufacturer's protocol (Becton Dickinson) and stained with fluorescent-conjugated CD3 (Invitrogen), CD4, CD25, CD56 (BD Biosciences), and pSTAT5 (eBioscience) antibodies. Cells were analyzed by flow cytometry (NovoCyte model 3000 and NovoExpress software, Acea). IL-21 stimulated cells were stained with antibodies for CD4, CD8, and CD19 (BioLegend) for $20 \mathrm{~min}$ on ice and fixed with $4 \%$ formaldehyde (Thermo) for $10 \mathrm{~min}$. After washing, cells were

d
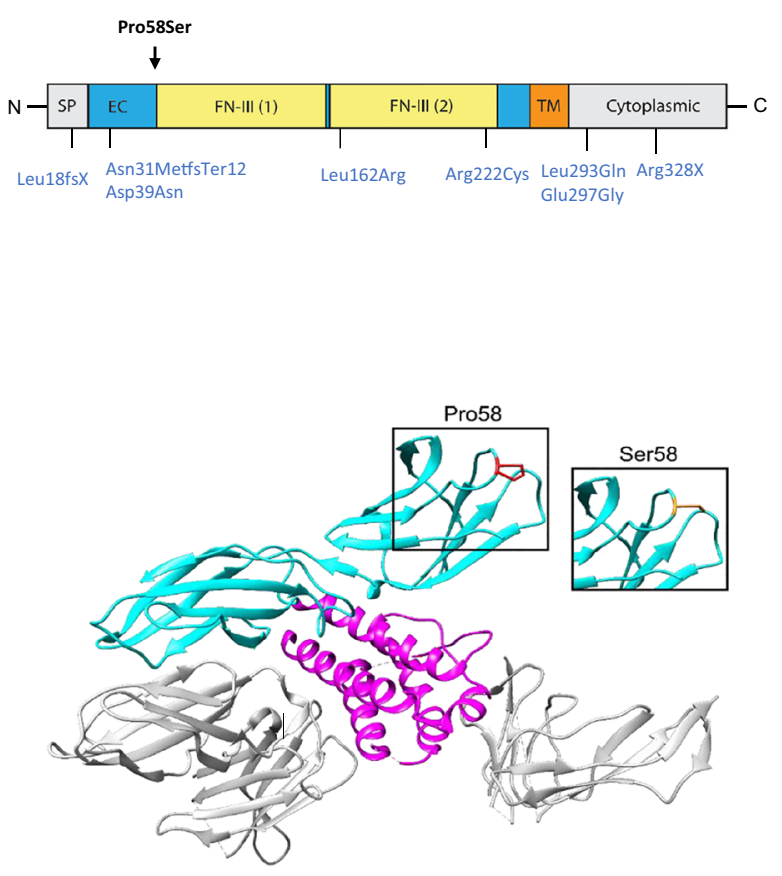

reported hypomorphic $I L 2 R G$ mutations denoted (in blue). Signal peptide (SP: positions 1-22) and domains extracellular (EC: 23-262), fibronectin type III (FN-III): (1): 59-151; (2):154-2462, transmembrane (TM: 263-283) and cytoplasmic: (284-369) (based on NCBI Reference Sequence: NP_000197.1 and UniProtKB- P31785). d Structure of Homo sapiens IL-2 cytokine receptor complex (Protein Data Bank accession number $2 \mathrm{~b} 5 \mathrm{i}$ ). Complex contains 4 protein chains; IL-2 (magenta), IL2RG (cyan), and IL2RA and IL2RB (both gray). The Pro58 residue in IL2RG highlighted in red and Ser58 mutation in orange

incubated in $90 \%$ methanol at $-20{ }^{\circ} \mathrm{C}$ overnight and stained with fluorescent conjugated anti-CD3 (BD), anti-pSTAT3 (Y705, BD), and anti-STAT3 (BD). Cells were analyzed using BD Fortessa flow cytometer and FloJo (v10.6) software.

\section{Quantitative PCR}

CD4+ cells were purified from PBMCs by negative selection with the human CD4+ isolation kit (Miltenyi Biotec) according to the manufacturer's instructions. Cells were incubated in RPMI 1640 medium (Gibco, Thermo Fischer Scientific) supplemented with $0.5 \%$ FBS (HyClone), $2 \mathrm{mM} \mathrm{L-glutamine} \mathrm{and}$ $0.1 \mathrm{mg} / \mathrm{ml}$ penicillin-streptomycin (Gibco, Thermo Fischer Scientific); $10^{6}$ cells/well at $+37{ }^{\circ} \mathrm{C}$ for $2 \mathrm{~h}$ prior to IL-2 stimulation (100 U/ml, Sigma). After 4-h stimulation, cells were pelleted and snap-frozen to be further processed. Both stimulated and unstimulated conditions were performed in triplicates for each sample. RNA was extracted using Qiagen RNeasy Mini kit according to the manufacturer's instructions. A total of $240 \mathrm{ng}$ of RNA was used as a template for cDNA synthesis (Bio-Rad iScript cDNA synthesis kit according to the manufacturer's instructions). Quantitative PCR (qPCR) in technical triplicates was performed using Taqman protocol 
and probes according to the manufacturer's instructions and analyzed with BioRad CFX Touch Real-Time system. The used Taqman probes are listed in the Online Resource Supplementary text. Results were analyzed with GraphPad Prism 7.03 software.

\section{Blast Formation and Lymphocyte Proliferation in Response to Exogenous IL-2}

Blast formation was studied by flow-cytometric assay for specific cell-mediated immune-responses in activated whole blood (FASCIA). Heparinized blood was diluted in RPMI medium containing Glutamax I, gentamicin, and $\beta$ mercaptoethanol and stimulated with IL-2 (R\&D Systems) in concentrations of $40 \mathrm{U} / \mathrm{ml}$ and $320 \mathrm{U} / \mathrm{ml}$ for 5 days. After stimulation, cells were stained with Multitest 6-Color TBNK reagent (BD) including $\mathrm{CD} 3, \mathrm{CD} 45, \mathrm{CD} 4, \mathrm{CD} 8, \mathrm{CD} 19$, and CD16/CD56 antibodies. After red blood cells were lysed with FACS lysing solution (BD), lymphocyte subpopulations were analyzed for the percentage of blasts based on their light scatter characteristics using flow cytometry (NovoCyte; Acea). In studying lymphocyte proliferation, purified PBMCs were incubated in culture medium $\left(2 \times 10^{5}\right.$ cells per well $)$ in 96-well polystyrene plates in the presence of increasing concentrations of IL-2 (R\&D Systems) for $72 \mathrm{~h}$. For the last $6 \mathrm{~h}, 1 \mu \mathrm{Ci}$ of $3 \mathrm{H}-$ Thymidine was added to each well. All conditions were performed in triplicates. Cells were harvested using a cell harvester (Tomtec) and thymidine incorporation was measured with beta-scintillation counter (MicroBeta; Perkin Elmer).

\section{IL2RG Expressing Flp-In 293T-REx Cell Lines}

Pro58Ser-mutant plasmid was created using Quick-Change Site-Directed Mutagenesis kit (Agilent Technologies) to wild-type $I L 2 R G$ obtained from the human ORFeome collection. Constructs were further subcloned into C-terminal MAC-tag gateway destination vector [22]. The Flp-In 293TREx cells (Invitrogen) were cultured as instructed by the manufacturer. The constructs were transfected into cells with FuGENE HD Transfection Reagent (Promega) together with pOG44 Flp recombinase expression vector (Thermo Fisher Scientific). The cells were grown under selection with hygromycin B to create a stable cell line.

\section{Affinity Purification and Mass Spectrometry}

Affinity purification of BioID and liquid chromatographymass spectrometry (LC-MS), analyses were performed as previously described [22]. In BioID-method, a modified biotin ligase is fused to the protein of interest, which enables the biotinylation of the proteins that are proximal. The biotinylated closely interacting proteins are then affinity-purified and analyzed with quantitative mass spectrometry [23]. Briefly, for each pull-down, approximately $5 \times 10^{7}$ cells $(5 \times 15 \mathrm{~cm}$ dishes) in two biological replicates were induced with $2 \mu \mathrm{g} / \mathrm{ml}$ tetracycline and $50 \mu \mathrm{M}$ biotin (Sigma) for $24 \mathrm{~h}$. Cells were harvested, pelleted, and lysed in HENN-lysis buffer (HENN; 50 mM HEPES pH 8.0, 5 mM EDTA, $150 \mathrm{mM}$ $\mathrm{NaCl}, 50 \mathrm{mM} \mathrm{NaF}$, supplemented with $0.5 \% \mathrm{NP} 40,1 \mathrm{mM}$ DTT, $1.5 \mathrm{mM} \mathrm{Na}_{3} \mathrm{VO}_{4}, 1 \mathrm{mM}$ PMSF, and 1x protease inhibitors cocktail; Sigma). Affinity purification was done using Strep-Tactin beads (IBA GmbH). Bound proteins were eluted with freshly prepared D-biotin (Thermo Fisher Scientific). The sample proteins were reduced with TCEP (Tris(2carboxyethyl)phosphine), alkylated with iodoacetamide, and digested with trypsin (Promega).

LC-MS analysis was done with a Q Exactive ESIquadrupole-orbitrap mass spectrometer coupled to an EASYnLC 1000 nanoflow LC (Thermo Fisher Scientific) using the Xcalibur version 3.1.66.10 (Thermo Fisher Scientific). For each sample, two technical replicates were analyzed.

\section{Database Analysis}

SEQUEST search algorithm in Proteome Discoverer software (Thermo Fisher Scientific) was used for peak extraction and protein identification with the human reference proteome of UniProtKB database (www.uniprot.org, Uniprot human 10 2016, with 20,118 sequences). Allowed error tolerances were $15 \mathrm{ppm}$ and $0.05 \mathrm{Da}$ for the precursor and fragment ions, respectively. Database searches were limited to fully tryptic peptides allowing two missed cleavage and carbamidomethyl $+57,021 \mathrm{Da}(\mathrm{C})$ of cysteine residue was set as fixed, and oxidation of methionine $+15,995 \mathrm{Da}(\mathrm{M})$ as dynamic modification. For peptide identification, false discovery rate was set to $<0.05$. The high-confidence protein-protein interactions were identified using stringent filtering against green fluorescent protein control samples. The bait normalized relative protein abundances (\% to the IL2RG) were calculated from the spectral counts. Statistical difference was calculated with Student's $t$ test. Gene ontology (GO) annotations were obtained from DAVID bioinformatics resources [24, 25].

\section{Results}

\section{Case Report}

An 11-year-old boy with normal (-1SD) growth was born to nonconsanguineous Finnish parents (Fig.1a). At age two, he started having recurrent upper and lower respiratory tract infections, prolonged cough, and bilateral purulent middle ear infections. At age six, tympanostomy was performed. However, recurrent middle ear infection and purulent discharge from tympanostomy tubes continued and bronchiectasis was noted (Fig.1b), with cystic fibrosis and ciliary 
dyskinesia ruled out. After two bouts of post-infectious synovitis in the knee at ages eight and nine, HLA-B27, rheumatoid factor, cyclic citrullinated peptide antibodies, and antinuclear antibodies were tested negative.

The patient's T, B, and NK cell counts were within the normal range, with elevated $\gamma \delta$ lymphocyte counts comprising $35.9 \%$ of CD3+ T cells. Both CD4+ and CD8+ effector memory (TEM) populations were decreased, with almost absent CD4+ terminally differentiated effector cells (TEMRAs). Regulatory $T$ cell $\left(T_{\text {reg }}\right)$ numbers were normal. Numbers of circulating CD27+ B memory and switched memory B cells were very low. Plasmacytoid dendritic cells (DCs) comprised only $0.02 \%$ of the total leukocytes (Table S1, Online Resource). NK cell phenotyping showed that patient had increased amount of CD3-CD56 $6^{\text {bright }} \mathrm{NK}$ cells when compared to healthy controls. Furthermore, these cells expressed highly CD27 and inhibitory receptor NKG2A, while CD57 expression was lower than in controls (Table S2, Online Resource). TCRV $\beta$ repertoire was comparable to healthy controls (Table S3, Online Resource).

The patient mounted normal antibody responses to tetanus and pneumococcal vaccines, antibodies against Haemophilus influenzae and diphtheria reached protective levels only after additional booster doses. Proliferative responses to mitogens (concanavalin A, phytohemagglutinin, pokeweed) have fluctuated (Table S1, Online Resource).

Patient's plasma immunoglobulin levels have remained normal. Due to recurrent infections and bronchiectasis, azithromycin prophylaxis, and inhalations with hypertonic saline, fluticasone and salbutamol were started at age seven. As recurrent infections continued, intravenous immunoglobulin (IVIG) treatment was started at age nine. The frequency of infections reduced without evident progression of bronchiectasis or his clinical condition. For more detailed case report, see Supplementary text, Online Resource.

\section{Genetic Analysis}

WES identified a novel c. $172 \mathrm{C}>\mathrm{T}$ hemizygous missense variant in the IL2RG gene (ENSG00000147168:ENST00000374202: c. $172 \mathrm{C}>\mathrm{T}$;p.(Pro58Ser)). Proline-58 is conserved and located in the extracellular part of the IL2RG, close to the fibronectin-III domain (Fig. 1c, d). The screening of the patient's mother implicated a de novo mutation (Fig. 1a and S1, Online Resource). Analysis of the WES data did not reveal any other compelling pathogenic or likely pathogenic variants in genes that could contribute to the phenotype based on current knowledge (Tables S4 and S5, Online Resource). Sequencing of the patient's different lymphocyte subsets (CD4+, CD8+, CD19+) and CD16+CD56+ NK cells confirmed the presence of the mutation in all these (Fig.S1, Online Resource).

\section{Reduced IL2RG Expression on Cell Surface Affects IL-2, IL-15, and IL-21-Mediated Signal Transduction}

In order to study if the Pro58Ser amino acid substitution in IL2RG affects IL-2R expression on patient lymphocytes, we stained patient's and healthy donors' lymphocytes with antibodies specific for IL2RG (CD132) and IL2RA (CD25) and analyzed their surface expression by flow cytometry. In the patient, we found a clear reduction in both IL2RG and IL2RA surface expressions on CD4+ $\mathrm{T}$ cells when compared to healthy donors (Fig. 2a, b). Furthermore, we observed similar trends of lower IL2RG surface expression in patient's CD8+ T and CD56+ NK cells (Table S6, Online Resource). In order to study whether the reduced surface expression was due to lower $I L 2 R G$ gene in expression in patient cells, we performed qPCR on CD4+ T cells. The expression of $I L 2 R G$ mRNA in the patient's CD4+ T cells was at an equal or slightly increased level compared to our healthy controls (Fig.S2, Online Resource), indicating that Pro58Ser mutation may affect protein stability or targeting to cell surface.

To study whether signaling through IL-2R was impaired due to the mutated $I L 2 R G$, we studied STAT5 phosphorylation in response to IL-2. At low IL-2 concentrations (10 U/ml), STAT5 phosphorylation was evident on $5.6 \%$ of patient CD4+ T cells compared with $11.9 \%$ and $11.6 \%$ (mean $11.8 \%$ ) of CD4+ T cells in the controls (Fig. 2c). To assess if higher IL-2 concentration can compensate the signaling defect, we simulated patient and healthy control cells with $320 \mathrm{U} / \mathrm{ml}$ of IL-2, which induced STAT5 phosphorylation in $53.1 \%$ of CD4+ T cells in the study subject, compared to STAT5 phosphorylation in $80.7 \%$ and $81.4 \%$ (mean $70.3 \%$ ) of CD4+ T cells in healthy controls (Fig. 2d). Data indicate that the remaining IL-2R on the patient's CD4+ T cell surface was insufficient to mediate proper IL-2-induced STAT5 phosphorylation.

Next, we studied if reduced STAT5 phosphorylation affected the expression of known IL-2 target genes. After a 4-h stimulation with $100 \mathrm{U} / \mathrm{ml}$ IL-2, expression of $C D 25$ was significantly lower in the patient cells compared to healthy donors (approximately 4.5 -fold induction in the patient vs. 8 -fold induction in controls when compared to unstimulated conditions) (Fig. 2e). In response to IL-2 stimulation, leukemia inhibitory factor expression stayed at basal level in patient cells but increased significantly in controls (Fig. 2f).

With FASCIA, lymphocyte blast formation in response to varying IL-2 concentrations $(40 \mathrm{U} / \mathrm{ml}$ and $320 \mathrm{U} / \mathrm{ml})$ was clearly impaired in index patient's T (Fig. $2 \mathrm{~g}, \mathrm{~h}$ ) and NK (Fig. 2i) cells compared to healthy controls. His PBMCs also showed very weak proliferation responses to low concentrations of IL-2 (3 to $13 \%$ of the level in healthy controls at $10 \mathrm{U} /$ $\mathrm{ml}$ ) (Fig. 2j). At higher IL-2 concentrations (up to $320 \mathrm{U} / \mathrm{ml}$ ), an increasing proliferation response was detected in his cells (Fig. 2j) but remained lower than in controls (21 to $58 \%$ of the 

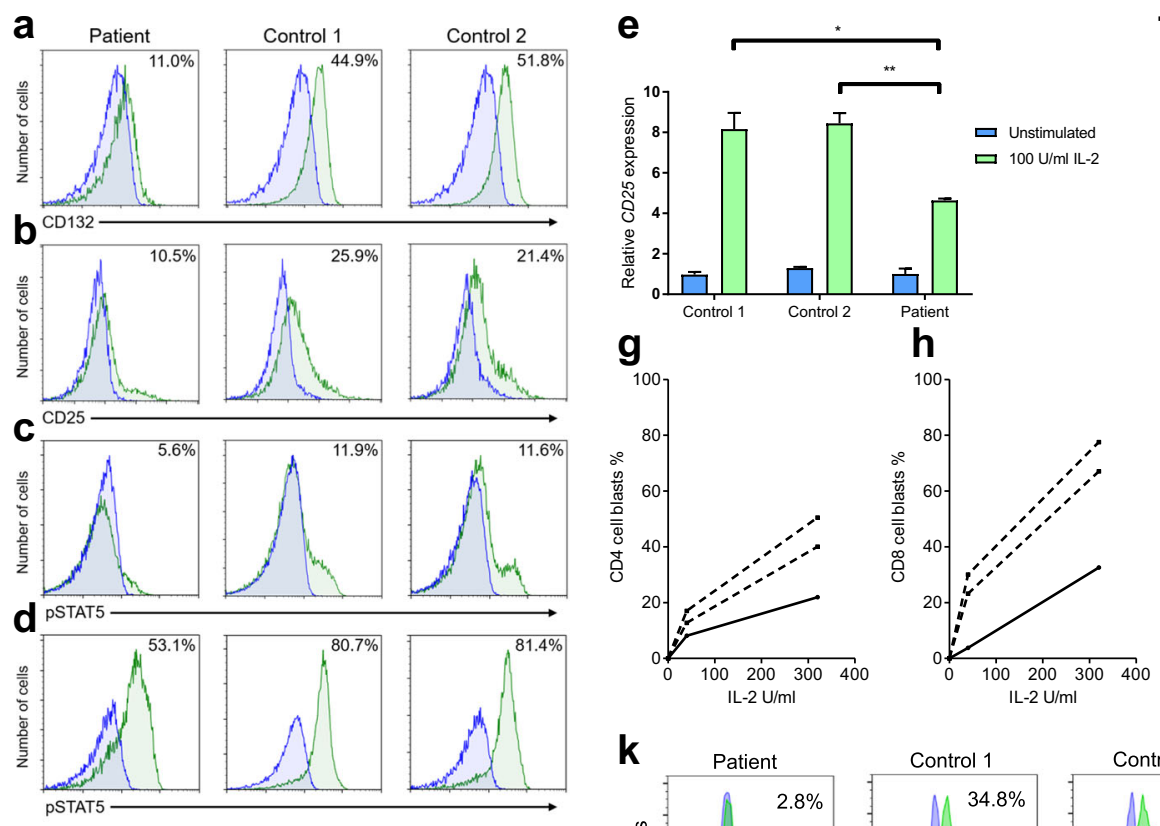

f
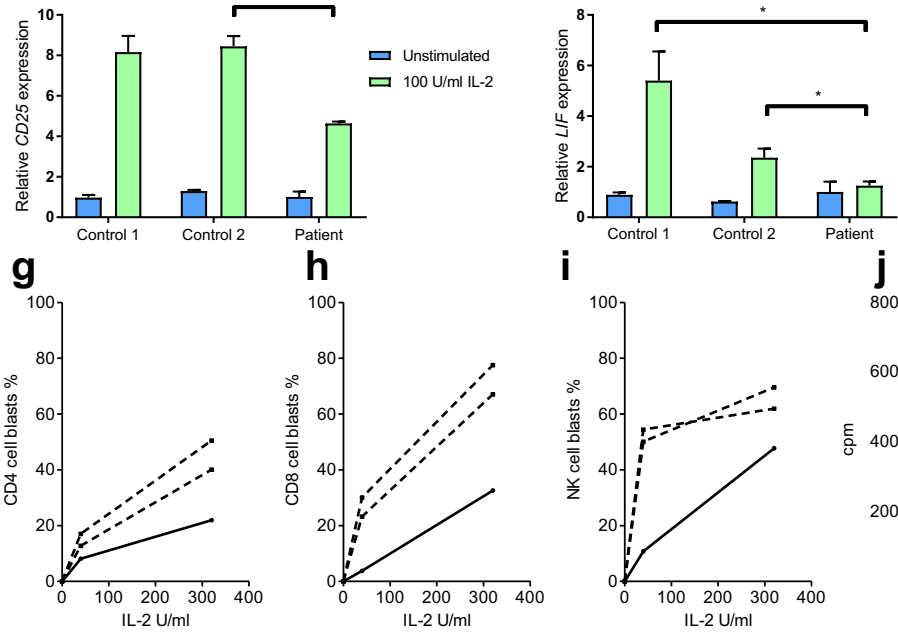

Unstimulated 口 $100 \mathrm{U} / \mathrm{ml} \mathrm{IL}-2$

i
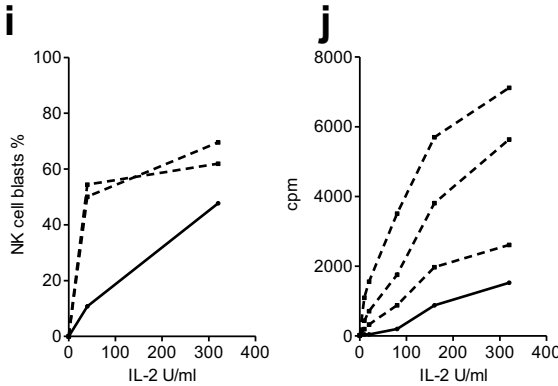

k

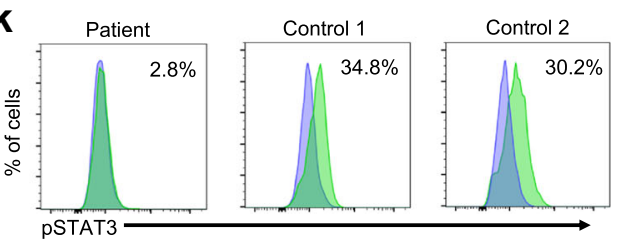

Fig. 2 IL-2 and IL-21 responses are disturbed in patient lymphocytes. Expression of a IL2RG (CD132) and b IL2RA (CD25) on patient's CD4+ T cells, and phosphorylation of STAT5 in CD4+ T cells in response to IL-2 stimulation at $\mathbf{c}$ low $(10 \mathrm{U} / \mathrm{ml})$ and $\mathbf{d}$ high $(320 \mathrm{U} / \mathrm{ml})$ concentration. Representative histograms for the patient (left) and two control subjects (middle, right) are shown in green as an overlay together with FMO staining (blue). e $C D 25$ and $\mathbf{f}$ leukemia inhibitory factor $(L I F)$ mRNA expression after 4-h stimulation with IL-2 (100 IU/ $\mathrm{ml}$ ) determined by quantitative PCR. Represented as fold change (patient unstimulated condition normalized as 1$)$. $\mathbf{g}$ CD4+ T cell, $\mathbf{h}$ CD8+ T cell, and $\mathbf{i}$ CD56+ NK cell blast formation in response to $40 \mathrm{U} / \mathrm{ml}$ and $320 \mathrm{U} /$

$\mathrm{ml}$ of IL-2 by FASCIA. $\mathbf{j}$ In vitro lymphocyte proliferation in response to various concentrations of IL-2. PBMC were stimulated for $72 \mathrm{~h}$ with 6 different concentrations of recombinant IL-2 in a $3 \mathrm{H}$-Thymidine incorporation assay. Curves represent proliferation (in cpm) of patient's cells (solid line) and of three healthy controls (dotted lines) all tested in parallel. $\mathbf{k}$ Phosphorylation of STAT3 in CD3-CD19+ B cells in response to IL-21 $(10 \mathrm{ng} / \mathrm{ml})$ shown in green as an overlay with unstimulated cells (blue). Data are representative out of three independent experiments in $\mathbf{a}-$ d, and out of two in the rest. * $=p<0.05 ; * *=p<0.01$, determined by unpaired $t$ test with Welch's correction in $\mathbf{e}-\mathbf{f}$

level in healthy controls at $320 \mathrm{U} / \mathrm{ml}$ of IL-2). With hypomorphic mutations of IL2RG, higher IL-2 concentrations could theoretically enhance signaling through IL-2R. However, compared to controls, high IL-2 concentrations had no relative effect on the STAT5 phosphorylation or proliferation. These data indicate that signaling through IL-2 receptor was impaired in patient lymphocytes.

Since IL2RG is also a component of IL4, IL-15, and IL-21 receptors, signal transduction for these cytokines was also studied [4]. We found that STAT3 phosphorylation in patient's B cells was clearly reduced in response to $10 \mathrm{ng} / \mathrm{ml}$ of IL-21 stimulation, compared to healthy controls (Fig. 2k). However, in T cells, we observed insignificant differences in STAT3 phosphorylation between the patient and controls (data not shown). An significant trend towards lower STAT6 phosphorylation in response to IL-4 stimulation in patient CD4+ T cells was noted, but it was normal in CD8+ T and CD19+ B cells (data not shown). STAT5 phosphorylation in response to IL15 was normal in patient's lymphocytes (data not shown). In

studying IL-15 responses by FASCIA, NK cells behaved normally, whereas blasting of CD4+ and CD8+ T cells was reduced (Fig. S3, Online Resource).

\section{Pro58Ser-Mutation Disturbs IL2RG Plasma Membrane Targeting}

To characterize why the Pro58Ser mutation affects the cell surface expression of IL2RG, we analyzed the interaction profiles of wild-type (WT) IL2RG and Pro58Ser IL2RG in inducibly expressing HEK293 cell lines using BioID proximity labeling [22]. BioID-analysis showed 69 significantly $(p<0.05)$ altered protein-protein interactions between Pro58Ser-mutant and WT IL2RG (Table S7, Online Resource). Localization-based classification of these altered interacting proteins revealed a marked change in IL2RG localization; mutant IL2RG had increased interactions with ER/ Golgi and nuclear proteins, whereas interactions with the proteins localized in focal adhesion were clearly diminished 
Fig. 3 Pro58Ser IL2RG mutant displays increased interactions with ER/Golgi and nuclear proteins. BioID analysis of Pro58Ser mutant- and WT IL2RG. a Interacting proteins with a significant differences $(p<0.05)$ between Pro58Sermutant and WT IL2RG. The mean fold changes are calculated as label-free MS/MS abundances of Pro58Ser/WT IL2RG. The interactors are classified based on cellular localization. Red dashed line indicates more than two times change on the interacting protein abundance in Pro58Ser mutant compared with the wild type. FA $=$ focal adhesion; star $=$ no detected in WT IL2RG (on/offdifferences). b Interacting proteins that differ between Pro58Ser and WT were categorized according to their involvement in biological processes (Gene Oncology, Biological Processes terms) via DAVID bioinformatics resources

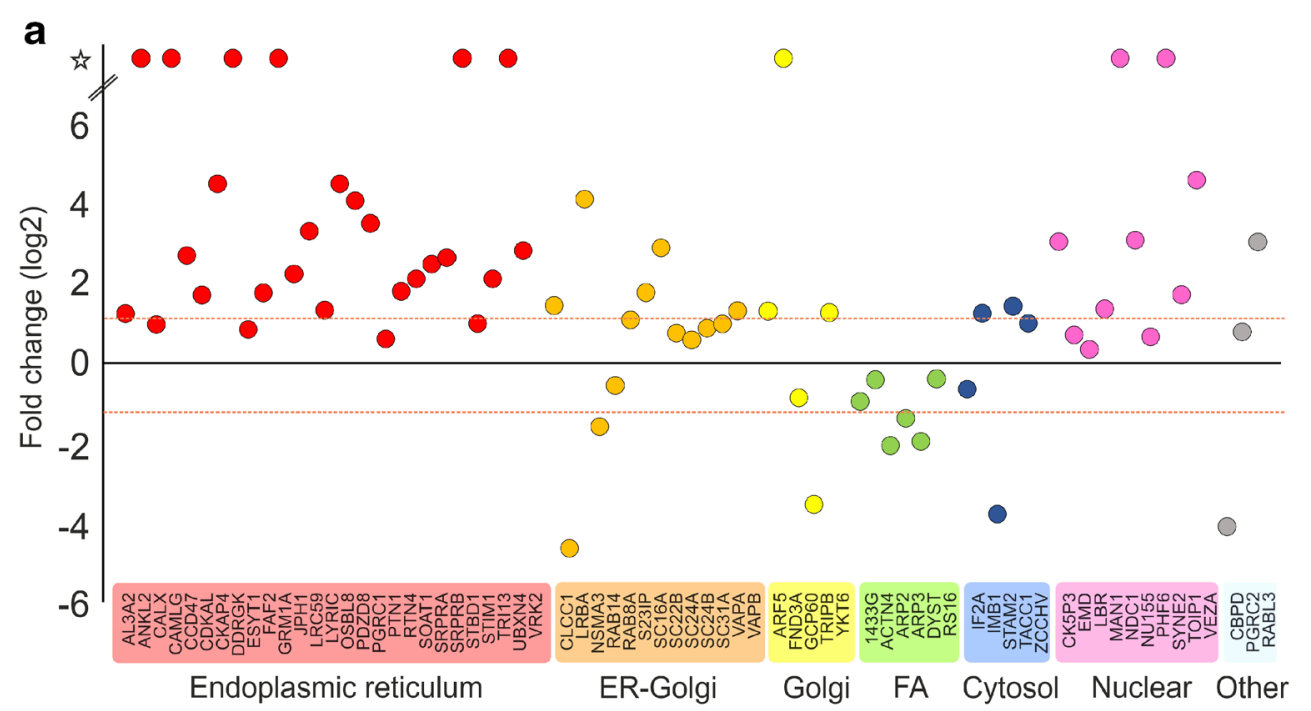

b

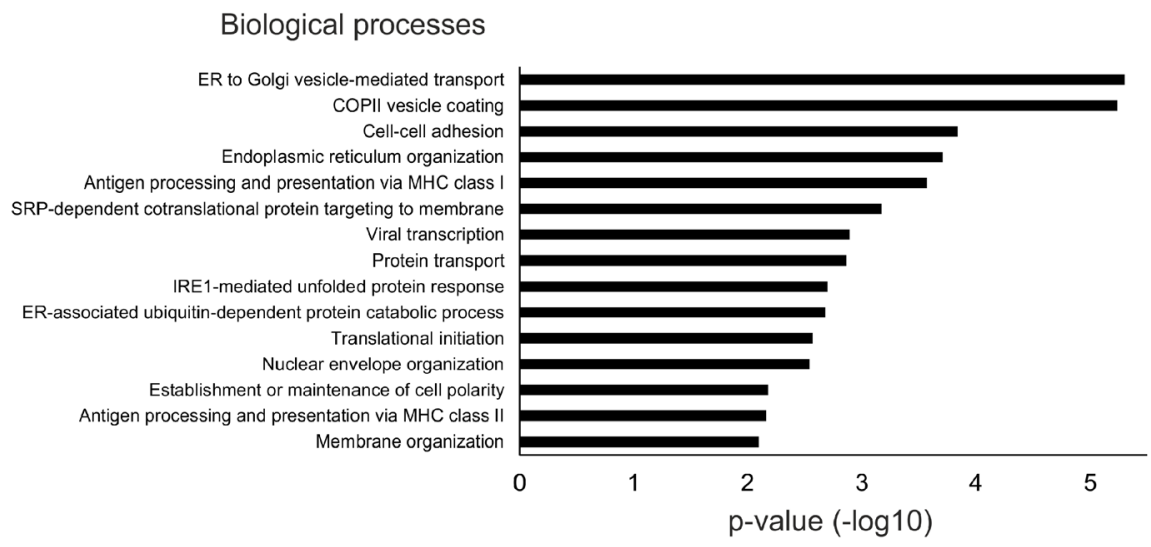

(Fig. 3a). In addition, a GO analysis for their biological processes showed that mutation significantly increased interactions with proteins involved in many ER-Golgi-associated functions, such as ER to Golgi vesicle-mediated transport and COPII vesicle coating (Fig. 3b). These results suggest abnormal maturation of mutant IL2RG, which causes the failure of the plasma membrane targeting and the mislocalization of mutant IL2RG to the ER-Golgi interface.

\section{Discussion}

We report a novel IL2RG p.(Pro58Ser) mutation causing XCID and provide evidence for the impaired IL-2 responses seen in the patient being caused by increased retention of the mutated protein in ER and Golgi. Our patient's CD4+ cells, compared to healthy controls, showed reduced surface expression of IL2RG and IL2RA (CD25), leading to impaired STAT phosphorylation responses to IL-2 and IL-21 (Fig. 2). Reduced or absent cell surface expression of IL2RG, caused by defect in the transcriptional level or by protein instability, has been reported in hypomorphic IL2RG mutations [13, 26-30]. Direct effect of IL2RG mutation on CD25 surface expression has been previously demonstrated in EBVtransformed cell lines [31] and CD34+ hematopoietic progenitor cells [32] of X-SCID patients along with one additional case termed as atypical X-SCID in which both absolute CD3+ counts and mitogen responses fluctuated so that unambiguous categorization was rendered impossible [33]. The altered amino acid residue resides in the extracellular domain of IL2RG (Fig. 1c, d); theoretically, this could also affect its ligandbinding affinity, but this remains to be studied.

During the revision process of this article, another boy detected by newborn screening for SCID with identical mutation came to our attention. He had low but not undetectable amounts of TRECs and presented with moderately decreased CD4+ and normal B, CD8+ and NK cell counts (Table S8, Online Resource). He is currently 3 years old without signs of illness. A patient with late-onset CID and probable revertant somatic mosaicism with a different mutation in the same 
position (c. 172C>A, p.(Pro58Thr)) has also been reported [12]. This patient had decreased IgA and IgG levels, occasional swelling of the knee and elbow joints, recurrent interstitial pneumonia, and watery diarrhea. He died of graft failure after an unsuccessful hematopoietic stem cell transplantation (HSCT).

The high-affinity heterotrimeric IL-2R receptor consists of IL2RA (CD25), IL2RB (CD122), and IL2RG (CD132) [34].

Reduced expression of any of the receptor components may affect IL-2 signaling [35-37]. IL2RG participates in signaling of interleukins 2, 4, 7, 9, 15, and 21 [4-7]. Impact of IL2RG mutations on various IL2RG-dependent pathways varies; hierarchies in the cytokine signaling impairment have been shown with some IL2RG-dependent signaling pathways more or less impaired than others $[26,29,38,39]$. In our patient, IL-2 and IL-15 signaling in T and IL-21 signaling in B cells were impaired, while IL-4 signaling was insignificantly affected (Fig. $2 \mathrm{c}-\mathrm{k}$ and Fig. S3b-c, Online Resource). Like in the boy caught by SCID screening, hypomorphic $I L 2 R G$ mutations frequently present with preserved or mildly decreased T and NK cells [27, 39-41]. The moderate functional defects and normal numbers of T, B, and NK cells in the patient also suggest partly retained signaling (Table S1). IL-2 is required for the sustained expansion of $\mathrm{T}$ cell populations and promotes the proliferation and survival of T cell receptor (TCR)-activated T cells [6]. It influences effector $\mathrm{T}$ cell differentiation and promotes fate decisions in antigen receptor-activated T cells [34]. Reflecting disturbed IL-2 signaling, our patients displayed reduced cell proliferation in response to IL-2 and low numbers of CD4+ TEMs and TEMRAs together with low CD8+ TEMs and T central memory cells (Fig. 2j, Tables S1, 8, Online Resource). IL-2 is also critical for the development of $\mathrm{T}_{\text {regs }}$ in the thymus and for their maintenance and function in the periphery; index patient's normal numbers of $T_{\text {regs }}$ and the lacking severe autoimmunity further confirmed hypomorphism (Table S1).

IL-21 and IL-4 are produced and secreted by T follicular helper cells $\left(\mathrm{T}_{\mathrm{FH}}\right)[8,42]$. IL-21 supports the differentiation and survival of $\mathrm{B}$ cells and is required for germinal center reactions, production of plasma cells and IgG class switch recombination [43, 44]. IL-4, in autocrine fashion, enables $\mathrm{T}_{\mathrm{FH}} \mathrm{S}$ to activate the activation-induced cytidine deaminase enzyme in B cells, which in turn is necessary for classswitch recombination and affinity maturation [8]. Thus, IL-4 and IL-21 in combination are required for normal B cell function and humoral responses. Besides IL-4 and IL-21, plasma cell differentiation also requires IL-2 $[45,46]$. Our patient had high numbers of naïve B cells but low numbers of switched memory B cells, B memory cells, and plasmablasts, likely caused by defective IL2RG plasma membrane targeting and consequently impaired collaborative IL-2 and IL-21 signaling. The patient benefited from IVIG therapy despite apparently normal serum immunoglobulin levels. This may indicate that his endogenous immunoglobulins may not mature into full avidity and/or his B cell memory may not be long-lasting.

The patient also had very low number of plasmacytoid DCs, which could be associated with the defective IL2RG-dependent cytokine signaling; IL-4 and IL-15 are known survival factors for DCs [6]. Although the IL2RG expression was reduced on the surface of patient's NK cells, their level in peripheral blood has remained normal (Tables S1 and S6) [4, 34]. However, their IL-2-stimulated blast formation was impaired (Fig. $2 \mathrm{i}$ ), indicating that IL-2 signaling in patient's NK cells was partly affected by IL2RG Pro58Ser mutation. Patient had increased proportion of CD $56^{\text {bright }}$ NK cells, usually considered cytokine expressing immature cells able to become cytotoxic upon appropriate activation (Table S2, Online Resource) [47]. These cells expressed highly the effector marker CD27 (84.1\% of CD56 $6^{\text {bright }}$ cells) and the inhibitory receptor NKG2A $(71.5 \%$ of CD56 $6^{\text {bright }}$ cells) and decreased amounts of CD57, a marker for terminally matured NK cells $[48,49]$. In conclusion, patient's CD56 $6^{\text {bright }} \mathrm{NK}$ cells displayed characteristics of both early and later NK cell development stages. Similarly, increased frequencies of CD56 $6^{\text {bright }}$ NK cells have been reported in a patient with CD25 deficiency [50]. Like for the case with CD25 deficiency, our preliminary data suggest that the degranulation ability of both $\mathrm{CD} 56^{\text {briht }}$ and $\mathrm{CD} 56^{\mathrm{dim}}$ cells was restored when patient cells were stimulated with K562 cells in vitro (data not shown).

In increasing numbers of genetic immunodeficiencies, including hypomorphic SCID gene mutations, lymphocyte numbers remain normal despite clear functional defects [51]. Numbers of reported patients carrying such mutations have increased sharply with the increasing use of next-generation sequencing (NGS) early in the diagnostic process. The early use of NGS followed by functional testing of novel mutations enables expansion of the initially described, typically very severe phenotypes, and thus greatly facilitates the diagnosis and targeted treatment of CID patients. In Table 1, we briefly review the reported, putatively hypomorphic IL $2 R G$ mutations [11, 13, 26-29, 38-41, 52-55]. Atypical X-SCID cases are frequently also caused by genetic reversion [15-17, 19, 58], which can easily be detected by sequencing of different lymphocyte subpopulations. To exclude maternal engraftment, one may use HLA typing or short tandem repeat (STR) analysis $[18,59,60]$. Frequently in historical reports, somatic mosaicism and/or maternal T or NK cell engraftment have not been excluded systematically or the numbers of CD4+ and CD8+ subsets were provided without information of the overall $\mathrm{T}$ cell numbers or the numbers of $\gamma \delta \mathrm{T}$ cells [11, $26,38,39,53,55]$. In these cases, we considered the mutations as potentially hypomorphic and included them in our 


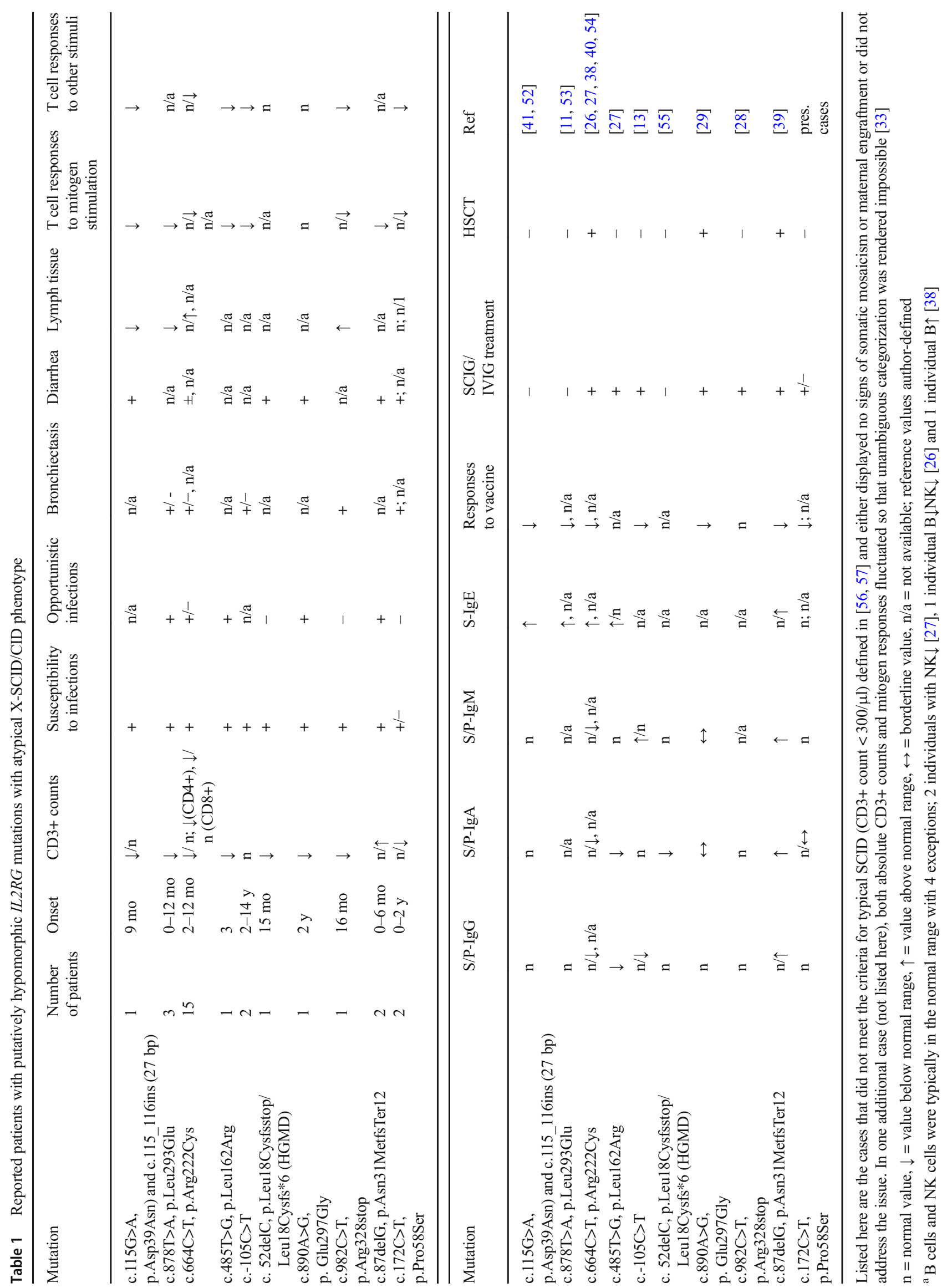


review. Of note, reactive expansion of the $\gamma \delta \mathrm{T}$ cells seems particularly common in atypical X-SCID patients [61].

A well-known example of hypomorphism in $I L 2 R G$ is the c. 664C >T;p.(Arg222Cys) mutation, so far reported in 18 patients $[26,27,38,40,54]$. In different reports, these patients were classified as typical or atypical, with the majority presenting with opportunistic infections or an Omenn-like clinical presentation; vast majority of them were treated with HSCT. Ten cases of Arg222Cys patients were included in Table 1 and eight excluded due to an X-SCID phenotype.

Altogether, 29 patients with atypical, putatively hypomorphic IL2RG mutations causing atypical X-SCID (variably named as X-CID) have been reported, including ours. Prevalence of the most common clinical features is presented in Fig. S4 (Online Resource). The most common feature (97\%) is susceptibility to infections, severest forms of viral and fungal infections were rare. However, 13 (45\%) patients had suffered from infections caused by opportunistic pathogens, four despite normal CD3+ T cell counts [27, 40]. Reported inflammatory conditions were surprisingly rare in reviewed patients carrying hypomorphic $I L 2 R G$ mutations, with only three patients suffering from eczema or other skin rashes [11, 26, 38], two from inflammatory arthritis [39], two from interstitial lung disease [38], and one from inflammatory bowel disease [29].

Including our patients, 12 (41\%) patients presented with normal plasma/serum immunoglobulin levels (IgG normal in all; IgM, $\operatorname{IgA}$ and IgE variable). Interestingly, eight (28\%) of the reviewed cases showed similarly skewed B cell subpopulations with increased numbers of naïve cells and/or decreased switched memory cells and plasmablasts as our index patient [26, 29, 38, 39]. Despite constantly normal immunoglobulin levels, our index patient's clinical condition improved significantly after the start of IVIG treatment. Of the reviewed patients, $16(55 \%)$ had received HSCT and 10 (34\%) immunoglobulin replacement, some prior to HSCT. Currently, choosing treatment for X-CID is difficult. Long-term complications and prognosis of combined immunodeficiencies remain unknown. In the future, X-CID patients should be recruited to international registries and follow-up studies to collect information in order to outline treatment options and protocols.

Acknowledgments We thank the patients, their families, and healthy donors for their support. We thank Dr. Nina Peitsaro (Helsinki University flow cytometry unit) for sorting service. Eira Leinonen is acknowledged for help with sample collection. We thank Ms. Hanna Lähteenmäki (University of Helsinki) for NK-cell phenotyping experiment. We thank Dr. Petteri Arstila (Univ. of Helsinki) for CD3, CD14, CD16, and CD56 antibodies.

Authorship Contributions J.G., M.V., J.K., J.S., A.H., S.M., and M.S planned and supervised the project. E.T., J.G., R.T., S.P., L.T., A.K., and T.Ö. designed and performed experiments and analyzed the data. K.H., J.R., and J.V. provided clinical data. E.T., J.G., L.T., K.H., T.Ö., J.S., A.H., J.K., and M.S. participated in manuscript writing. All authors critically reviewed the manuscript.
Funding Information Open access funding provided by University of Helsinki including Helsinki University Central Hospital. Funding was received by Pediatric Academic Track funding (J.G.) and hospital research funds (E.T., M.S.) from Pediatric Research Center, HUS Helsinki University Hospital and by grants from Emil Aaltonen Foundation (J.G.), Academy of Finland (J.G., M.V., T.Ö., S.M.), Finnish Pediatric Research Foundation (E.T., J.G., M.S.), Sigrid Jusélius Foundation (J.K., M.V., T.Ö.), Novo Nordisk Foundation (A.H.), Finnish Cancer Institute (M.V., T.Ö.), Magnus Ehrnrooth Foundation (M.V., T.Ö.), and The Children's Research Institute, Milwaukee WI, USA (J.V.).

\section{Compliance with Ethical Standards}

Ethics Statement Blood samples from the patient and controls were collected after written informed consent, in accordance with the 1964 Declaration of Helsinki. The study protocols were approved by the Coordinating Ethics Committee of The Hospital District of Helsinki and Uusimaa (138/13/03/00/2013, HUS/26/2018) and the Ethics Committee for Gynaecology, Obstetrics, Pediatrics and Psychiatry (141/13/03/03/ 2012). This article does not contain any animal studies.

Conflict of Interest The authors declare that they have no conflict of interest.

Open Access This article is licensed under a Creative Commons Attribution 4.0 International License, which permits use, sharing, adaptation, distribution and reproduction in any medium or format, as long as you give appropriate credit to the original author(s) and the source, provide a link to the Creative Commons licence, and indicate if changes were made. The images or other third party material in this article are included in the article's Creative Commons licence, unless indicated otherwise in a credit line to the material. If material is not included in the article's Creative Commons licence and your intended use is not permitted by statutory regulation or exceeds the permitted use, you will need to obtain permission directly from the copyright holder. To view a copy of this licence, visit http://creativecommons.org/licenses/by/4.0/.

\section{References}

1. Kwan A, Abraham RS, Currier R, Brower A, Andruszewski K, Abbott JK, et al. Newborn screening for severe combined immunodeficiency in 11 screening programs in the United States. JAMA. 2014;312:729-38.

2. Notarangelo LD. Primary immunodeficiencies. J Allergy Clin Immunol. 2010;125:S182-94.

3. Fischer A. Severe combined immunodeficiencies (SCID). Clin Exp Immunol. 2000;122:143-9.

4. Malek TR. The biology of interleukin-2. Annu Rev Immunol. 2008;26:453-79.

5. Leonard WJ. Cytokines and immunodeficiency diseases. Nat Rev Immunol. 2001;1:200-8.

6. Rochman Y, Spolski R, Leonard WJ. New insights into the regulation of T cells by $\gamma$ c family cytokines. Nat Rev Immunol. 2009;9: 480.

7. Kovanen PE, Leonard WJ. Cytokines and immunodeficiency diseases: critical roles of the $\gamma \mathrm{c}$-dependent cytokines interleukins 2,4 , $7,9,15$, and 21 , and their signaling pathways. Immunol Rev. 2004;202:67-83.

8. Weinstein JS, Herman EI, Lainez B, Licona-Limón P, Esplugues E, Flavell R, et al. TFH cells progressively differentiate to regulate the germinal center response. Nat Immunol. 2016;17:1197-205. 
9. Plum J, De Smedt M, Leclercq G, Verhasselt B, Vandekerckhove B. Interleukin-7 is a critical growth factor in early human T-cell development. Blood. 1996;88:4239.

10. Roifman CM, Somech R, Kavadas F, Pires L, Nahum A, Dalal I, et al. Defining combined immunodeficiency. J Allergy Clin Immunol. 2012;130:177-83.

11. Brooks EG, Schmalstieg FC, Wirt DP, Rosenblatt HM, Adkins LT, Lookingbill DP, et al. A novel X-linked combined immunodeficiency disease. J Clin Invest. 1990;86:1623-31.

12. Okuno Y, Hoshino A, Muramatsu H, Kawashima N, Wang X, Yoshida $\mathrm{K}$, et al. Late-onset combined immunodeficiency with a novel IL2RG mutation and probable revertant somatic mosaicism. J Clin Immunol. 2015;35:610-4.

13. Chandra A, Zhang F, Gilmour KC, Webster D, Plagnol V, Kumararatne DS, et al. Common variable immunodeficiency and natural killer cell lymphopenia caused by Ets-binding site mutation in the IL-2 receptor $\gamma$ (IL2RG) gene promoter. J Allergy Clin Immunol. 2016;137:940-2.

14. Estévez OA, Ortega C, Fernández S. A novel IL2RG mutation presenting with atypical $\mathrm{T}(-) \mathrm{B}(+) \mathrm{NK}+$ phenotype: rapid elucidation of NK cell origin. Pediatr Blood Cancer. 2013;61:178-9.

15. Stephan V, Wahn V, Le Deist F, Dirksen U, Bröker B, MüllerFleckenstein I, et al. Atypical X-linked severe combined immunodeficiency due to possible spontaneous reversion of the genetic defect in T cells. N Engl J Med. 1996;335:1563-7.

16. Kawai T, Saito M, Nishikomori R, Yasumi T, Izawa K, Murakami $\mathrm{T}$, et al. Multiple reversions of an IL2RG mutation restore $\mathrm{T}$ cell function in an X-linked severe combined immunodeficiency patient. J Clin Immunol. 2012;32:690-7.

17. Hsu AP, Pittaluga S, Martinez B, Rump AP, Raffeld M, Uzel G, et al. IL2RG reversion event in a common lymphoid progenitor leads to delayed diagnosis and milder phenotype. J Clin Immunol. 2015;35:449-53.

18. Müller SM, Ege M, Pottharst A, Schulz AS, Schwarz K, Friedrich W. Transplacentally acquired maternal $\mathrm{T}$ lymphocytes in severe combined immunodeficiency: a study of 121 patients. Blood. 2001;98:1847.

19. Yamashita M, Wakatsuki R, Kato T, Okano T, Yamanishi S, Mayumi N, et al. A synonymous splice site mutation in IL2RG gene causes late-onset combined immunodeficiency. Int $\mathrm{J}$ Hematol. 2019;109:603-11.

20. Trotta L, Hautala T, Hämäläinen S, Syrjänen J, Viskari H, Almusa $\mathrm{H}$, et al. Enrichment of rare variants in population isolates: single AICDA mutation responsible for hyper-IgM syndrome type 2 in Finland. Eur J Hum Genet. 2016;24:1473-8.

21. Trotta L, Martelius T, Siitonen T, Hautala T, Hämäläinen S, Juntti $\mathrm{H}$, et al. ADA2 deficiency: clonal lymphoproliferation in a subset of patients. J Allergy Clin Immunol. 2018;141:1534-7.

22. Liu X, Salokas K, Tamene F, Jiu Y, Weldatsadik RG, Öhman T, et al. An AP-MS- and BioID-compatible MAC-tag enables comprehensive mapping of protein interactions and subcellular localizations. Nat Commun. 2018;9:1188.

23. Roux KJ, Kim DI, Raida M, Burke B. A promiscuous biotin ligase fusion protein identifies proximal and interacting proteins in mammalian cells. J Cell Biol. 2012;196:801.

24. Huang DW, Sherman BT, Tan Q, Kir J, Liu D, Bryant D, et al. DAVID bioinformatics resources: expanded annotation database and novel algorithms to better extract biology from large gene lists. Nucleic Acids Res. 2007;35:W169-75.

25. Huang DW, Sherman BT, Lempicki RA. Systematic and integrative analysis of large gene lists using DAVID bioinformatics resources. Nat Protoc. 2008;4:44.

26. Fuchs S, Rensing-Ehl A, Erlacher M, Vraetz T, Hartjes L, Janda A, et al. Patients with $\mathrm{T}+/$ low NK+ IL-2 receptor $\gamma$ chain deficiency have differentially-impaired cytokine signaling resulting in severe combined immunodeficiency. Eur J Immunol. 2014;44:3129-40.
27. Mella P, Imberti L, Brugnoni D, Pirovano S, Candotti F, Mazzolari E, et al. Development of autologous T lymphocytes in two males with X-linked severe combined immune deficiency: molecular and cellular characterization. Clin Immunol. 2000;95:39-50.

28. Lim CK, Abolhassani H, Appelberg SK, Sundin M, Hammarström L. IL2RG hypomorphic mutation: identification of a novel pathogenic mutation in exon 8 and a review of the literature. Allergy, Asthma Clin Immunol. 2019;15:2.

29. Neves JM, Cordeiro C, Ana I. Novel IL2RG mutation causes leaky TLOWB+NK+ SCID with nodular regenerative hyperplasia and normal IL-15 STAT5 phosphorylation. J Pediatr Hematol Oncol. 2018;41:328-33.

30. Disanto JP, Dautry-Varsat A, Certain S, Fischer A, de Saint Basile G. Interleukin-2 (IL-2) receptor $\gamma$ chain mutations in X-linked severe combined immunodeficiency disease result in the loss of highaffinity IL-2 receptor binding. Eur J Immunol. 1994;24:475-9.

31. Puck JM, Pepper AE, Henthorn PS, Candotti F, Isakov J, Whitwam $\mathrm{T}$, et al. Mutation analysis of IL2RG in human X-linked severe combined immunodeficiency. Blood. 1997;89:1968-77.

32. Cavazzana-Calvo M, Hacein-Bey S, de Saint BG, De Coene C, Selz F, Le Deist F, et al. Role of interleukin-2 (IL-2), IL-7, and IL-15 in natural killer cell differentiation from cord blood hematopoietic progenitor cells and from gamma $\mathrm{c}$ transduced severe combined immunodeficiency X1 bone marrow cells. Blood. 1996;88: 3901.

33. Ursini MV, Gaetaniello L, Ambrosio R, Matrecano E, Apicella AJ, Salerno MC, et al. Atypical X-linked SCID phenotype associated with growth hormone hyporesponsiveness. Clin Exp Immunol. 2002;129:502-9.

34. Ross SH, Cantrell DA. Signaling and function of interleukin-2 in T lymphocytes. Annu Rev Immunol. 2018;36:411-33.

35. Fernandez IZ, Baxter RM, Garcia-Perez J, Vendrame E, Ranganath $\mathrm{T}$, Kong DS, et al. A novel human IL2RB mutation results in T and NK cell-driven immune dysregulation. J Exp Med. 2019;216: 1255-67.

36. Zhang Z, Gothe F, Pennamen P, James JR, McDonald D, Mata CP, et al. Human interleukin-2 receptor $\beta$ mutations associated with defects in immunity and peripheral tolerance. J Exp Med. 2019;216:1311-27.

37. Vignoli M, Ciullini Mannurita S, Fioravanti A, Tumino M, Grassi A, Guariso G, et al. CD25 deficiency: a new conformational mutation prevents the receptor expression on cell surface. Clin Immunol. 2019;201:15-9.

38. Stepensky P, Keller B, Shamriz O, von Spee-Mayer C, Friedmann $\mathrm{D}$, Shadur B, et al. T+ NK+ IL-2 receptor $\gamma$ chain mutation: a challenging diagnosis of atypical severe combined immunodeficiency. J Clin Immunol. 2018;38:527-36.

39. Illig D, Navratil M, Kelečić J, Conca R, Hojsak I, Jadrešin O, et al. Alternative splicing rescues loss of common gamma chain function and results in IL-21R-like deficiency. J Clin Immunol. 2019;39: 207-15.

40. Somech R, Roifman CM. Mutation analysis should be performed to rule out $\gamma \mathrm{c}$ deficiency in children with functional severe combined immune deficiency despite apparently normal immunologic tests. J Pediatr. 2005;147:555-7.

41. de Saint-Basile G, Le Deist F, Caniglia M, Lebranchu Y, Griscelli C, Fischer A. Genetic study of a new X-linked recessive immunodeficiency syndrome. J Clin Invest. 1992;89:861-6.

42. McGuire HM, Vogelzang A, Warren J, Loetsch C, Natividad KD, Chan TD, et al. IL-21 and IL-4 collaborate to shape T-dependent antibody responses. J Immunol. 2015;195:5123-35.

43. Linterman MA, Beaton L, Yu D, Ramiscal RR, Srivastava M, Hogan JJ, et al. IL-21 acts directly on B cells to regulate Bcl-6 expression and germinal center responses. J Exp Med. 2010;207(2):353-63. 
44. Zotos D, Coquet JM, Zhang Y, Light A, D'Costa K, Kallies A, et al. IL-21 regulates germinal center B cell differentiation and proliferation through a B cell-intrinsic mechanism. J Exp Med. 2010;207: 365-78.

45. Hipp N, Symington H, Pastoret C, Caron G, Monvoisin C, Tarte K, et al. IL-2 imprints human naive B cell fate towards plasma cell through ERK/ELK1-mediated BACH2 repression. Nat Commun. 2017;8:1443.

46. Le Gallou S, Caron G, Delaloy C, Rossille D, Tarte K, Fest T. IL-2 requirement for human plasma cell generation: coupling differentiation and proliferation by enhancing MAPK-ERK signaling. $\mathrm{J}$ Immunol. 2012;189:161-73.

47. Michel T, Poli A, Cuapio A, Briquemont B, Iserentant G, Ollert M, et al. Human CD56 ${ }^{\text {bright }}$ NK cells: an update. J Immunol. 2016;196: 2923-31.

48. Hayakawa Y, Smyth MJ. CD27 dissects mature NK cells into two subsets with distinct responsiveness and migratory capacity. J Immunol. 2006;176:1517-24.

49. Mace EM, Orange JS. Emerging insights into human health and NK cell biology from the study of NK cell deficiencies. Immunol Rev. 2019;287:202-25.

50. Caldirola MS, Rodríguez Broggi MG, Gaillard MI, Bezrodnik L, Zwirner NW. Primary immunodeficiencies unravel the role of IL-2/ CD25/STAT5b in human natural killer cell maturation. Front Immunol. 2018;9:1429.

51. Notarangelo LD. Chapter four - combined immunodeficiencies with nonfunctional T lymphocytes. Adv Immunol. 2014;121: 121-90.

52. Disanto JP, Rieux-Laucat F, Dautry-Varsat A, Fischer A, de Saint Basile G. Defective human interleukin 2 receptor gamma chain in an atypical $\mathrm{X}$ chromosome linked severe combined immuonodeficiency with peripheral T cells. Proc Natl Acad Sci U S A. 1994;91:9466-70.

53. Schmalstieg FC, Leonard WJ, Noguchi M, Berg M, Rudloff HE, Denney RM, et al. Missense mutation in exon 7 of the common gamma chain gene causes a moderate form of $\mathrm{X}$-linked combined immunodeficiency. J Clin Invest. 1995;95:1169-73.
54. Sharfe N, Shahar M, Roifman CM. An interleukin-2 receptor gamma chain mutation with normal thymus morphology. J Clin Invest. 1997; 100:3036-43.

55. Mou W, He J, Chen X, Zhang H, Ren X, Wu X, et al. A novel deletion mutation in IL2RG gene results in X-linked severe combined immunodeficiency with an atypical phenotype. Immunogenetics. 2017;69:29-38.

56. Shearer WT, Dunn E, Notarangelo LD, Dvorak CC, Puck JM, Logan BR, et al. Establishing diagnostic criteria for severe combined immunodeficiency disease (SCID), leaky SCID, and Omenn syndrome: the primary immune deficiency treatment consortium experience. J Allergy Clin Immunol. 2014;133:1092-8.

57. Hannon WH, Abraham RS, Kobrynski L, Vogt RF, Adair O, Aznar $\mathrm{C}$, et al. Newborn blood spot screening for severe combined immunodeficiency by measurement of T-cell receptor excision circles; Approved Guideline, CLSI document NBS06-A. CLSI. 2013;33(4).

58. Speckmann C, Pannicke U, Wiech E, Schwarz K, Fisch P, Friedrich $\mathrm{W}$, et al. Clinical and immunologic consequences of a somatic reversion in a patient with $\mathrm{X}$-linked severe combined immunodeficiency. Blood. 2008;112:4090-7.

59. Liu C, Duffy B, Bednarski JJ, Calhoun C, Lay L, Rundblad B, et al. Maternal T-cell engraftment interferes with human leukocyte antigen typing in severe combined immunodeficiency. Am J Clin Pathol. 2016;145:251-7.

60. Wahlstrom J, Patel K, Eckhert E, Kong D, Horn B, Cowan MJ, et al. Transplacental maternal engraftment and posttransplantation graftversus-host disease in children with severe combined immunodeficiency. J Allergy Clin Immunol. 2017;139:628-33.

61. Tometten I, Felgentreff K, Hönig M, Hauck F, Albert MH, Niehues $\mathrm{T}$, et al. Increased proportions of $\gamma \delta \mathrm{T}$ lymphocytes in atypical SCID associate with disease manifestations. Clin Immunol. 2019;201:30-4.

Publisher's Note Springer Nature remains neutral with regard to jurisdictional claims in published maps and institutional affiliations. 